\title{
Análisis de validación y confiabilidad de Rúbrica Socioformativa en torno al concepto gestión del conocimiento en proyectos formativos
}

\section{Validation and reliability analysis of Socioformative Rubric around the concept of knowledge management in training projects}

\author{
ANICETO, Paula F. ${ }^{1}$ \\ LUNA, Josemanuel. $^{2}$ \\ RODRÍGUEZ, María de L. ${ }^{3}$

\section{Resumen} \\ Debido a la falta de instrumentos que evalúen el proceso de gestión del conocimiento durante el \\ desarrollo de proyectos formativos en escuelas de nivel superior en el área de Ingeniería, este \\ artículo analiza la información recabada durante el proceso de validación por juicio de expertos y \\ determina su confiabilidad a través de la aplicación de un instrumento de evaluación aplicado a un \\ grupo piloto, proponiendo una rúbrica socioformativa confiable y satisfactoria para ser aplicada en el \\ contexto descrito. \\ Palabras clave: evaluación, gestión del conocimiento, proyectos formativos, socioformación.
}

\begin{abstract}
Due to the absence of application of instruments to evaluate the knowledge management process through the development of formative projects in Universities in the engineering area. This paper describes the analysis of information collected during the validation process through experts' judgement and determines the reliability through the application of the instrument to a pilot group, placing a reliable and satisfactory socio-formative rubric applied in the described context. key words: evaluation, knowledge management, training projects, socioformation.
\end{abstract}

\section{Introducción}

En esta investigación se socializa el proceso de diseño y análisis estadístico llevado a cabo para validar el contenido y determinar la confiabilidad de una rúbrica socioformativa como instrumento para evaluar el proceso de gestión del conocimiento durante el desarrollo de proyectos formativos en escuelas de Ingeniería. Un instrumento de evaluación debe reunir dos criterios de calidad: validez de contenido y fiabilidad. La validez considera dos aspectos: el diseño del instrumento para que "mida lo que debe medir" y la validación por un grupo de personas expertas que den fe de que efectivamente "sí mide lo que se quiere medir". El experto es

\footnotetext{
${ }^{1}$ Escuela Superior de Ingeniería Mecánica y Eléctrica Unidad Culhuaca, del IPN ( ESIME UC.). México. paniceto@ipn.mx

${ }^{2}$ Centro Universitario CIFE. México. josemanuelluna@cife.edu.mx

${ }^{3}$ Escuela Superior de Ingeniería Mecánica y Eléctrica Unidad Culhuaca, del IPN ( ESIME UC). México. mlrodriguezp@ipn.mx
} 
quien coadyuva a eliminar aspectos irrelevantes, valida los que son relevantes e incluso puede sugerir modificaciones, si así se requiere (Cabero-Almenara y Llorente-Cejudo, 2013). Escobar-Pérez y Cuervo Martínez (2008) consideran una persona experta aquella que puede dar información, evidencias, emitir juicios y valoraciones, debido a que tiene una trayectoria reconocida en el área de investigación y es reconocida por otros expertos calificados en el tema.

Ya varios autores han trabajado sobre la validez de contenido de un instrumento, por ejemplo, para JuárezHernández y Tobón (2018), la validez de contenido la refieren como la calidad y precisión de un instrumento de investigación y la relacionan con la presencia de demostraciones válidas y Haynes, Richard \& Kubany (1995) citado en Ibarra-Piza, et. al. (2018), consideran que el proceso de validez de contenido de un instrumento se puede hacer tanto por medio de estudios o métodos cualitativos, como cuantitativos. Así, también Urrutia, et. al. (2014), refieren a la validez de contenido como un juicio lógico en relación con una correspondencia entre las características de lo que se está evaluando con el aprendizaje del evaluado.

Haciendo referencia a los proyectos formativos desde la Socioformación, en ellos se articulan actividades con la finalidad de resolver problemas del contexto y así desarrollar las competencias del perfil de egreso de un programa académico (Hernández y Vizcarra, 2015). También considerados "como estrategias que la socioformación ha adapado de Kilpatrick (1918) para mediar el desarrollo del talento humano buscando que las personas se formen y vivan resolviendo problemas del contexto en un marco de trabajo colaborativo. Además, permite que las personas gestionen el saber en diferentes fuentes y construyan el conocimiento de manera participativa, con análisis", (Tobón, Cardona, Vélez Ramos, López Loya, 2015, p.21).

Para evaluar los proyectos formativos se considera relevante que el docente planifique la evaluación en donde implique la aplicación de instrumentos de evaluación con los que se valoren las evidencias y así poder determinar los niveles de desempeño y los errores cometidos durante un proceso de formación dirigidos hacia la mejora continua (Tobón, 2009; Hernández, Tobón y Ramírez, 2018). Al respecto, existe una gama de instrumentos que se pueden aplicar para evaluar las competencias en los proyectos formativos, por ejemplo; las listas de cotejo, las escalas de estimación, portafolio de evidencia, pero ante todo las rúbricas socioformativas (Cardona, Véles y Tobón (2015)(Mosqueda, Tobón, Antonio, 2015). Desde esta perspectiva, estas últimas, incorporan opciones para emitir juicios de evaluación de los desempeños que beneficien la mejora continua en las actuaciones de los estudiantes, ante los problemas de contexto partiendo de evidencias (Hernández - Mosqueda, Tobón y Guerrero - Rosas (2016).

Así también, estas tienen 4 características principales: 1) considera niveles de dominio; para la descripción de estos, se toma en cuenta la taxonomía socioformativa que aplica para ubicar los verbos de acuerdo a cada nivel de dominio: desde un nivel preformal, pasando por un nivel resolutivo hasta llegar al nivel estratégico; 2) se aplica a partir de un problema de contexto, este surge de una necesidad, un conflicto, un vacío; 3) se basa en una evidencia o producto central a evaluar, la rúbrica se diseña en función de este producto central; 4) considera la retroalimentación de manera integral para buscar la mejora continua (Tobón, 2017).

En escuelas de ingeniería, aún no se diseñan y aplican instrumentos para evaluar la gestión del conocimiento durante el desarrollo de proyectos formativos; por lo tanto, es considerado que las evaluaciones que se realizan al respecto son subjetivas. Los docentes no conocen el nivel de desempeño académico que los estudiantes tienen, solo emiten una calificación con un valor numérico en relación con el contenido temático de una asignatura o módulo. Hernández-Mosqueda, Tobón y Guerrero-Rosas (2016), consideran que en los países Iberoamericanos, incluyendo a México, las prácticas académicas que se llevan a cabo en las aulas están basadas aún en estrategias que se caracterizan por la transmisión de la información a los estudiantes, mismas prácticas descontextualizan la realidad; es por ello que es relevante atender este problema para lograr que las nuevas 
generaciones sean capaces de resolver problemas de diversas índoles a través del mejoramiento continuo de la apropiación del conocimiento.

Desde la socioformación, enfoque surgido en Iberoamérica, fundamentado en el constructivismo social y la epistemología de la complejidad (Tobón, 2013a), se hace relevante el hecho de proponer alternativas de solución, que permita a las autoridades, directivos, docentes, alumnos, familia y, en general, a las organizaciones sociales a trabajar con proyectos en donde se resuelvan problemas de contexto, con flexibilidad, ética y pertinencia (Tobón, 2011). Las competencias que debe tener un docente para tener facilidad de aplicar el aprendizaje basado en proyectos formativos son, en primer lugar, las competencias genéricas; en estas se localiza la mediación del aprendizaje y la evaluación de las competencias. En este caso, es necesario que el docente tenga el conocimiento para el diseño de actividades de aprendizaje relacionados con los propósitos de formación de las competencias, orientar a los estudiantes en el aprendizaje con relación a un módulo o unidad de aprendizaje o asignatura, generar ambientes propios para el aprendizaje acorde con las competencias a desarrollar.

En este documento se presenta el análisis de validación de contenido bajo el criterio de juicio de expertos y el contexto del enfoque socioformativo, así también la fiabilidad de la rúbrica socioformativa, para evaluar la apropiación de los aprendizajes como producto de la gestión del conocimiento; en donde el docente promueve la objetividad en la evaluación a través de la aplicación de instrumentos confiables, evaluación de los logros en los estudiantes para encaminarlos a la mejora continua y llegar a una formación integral. Lo anterior se hizo para satisfacer las necesidades de los estudiantes en el área de Ingeniería, que se apropien de los aprendizajes durante su formación profesional y los apliquen a lo largo de la vida. Por tal motivo, el presente estudio se enfocó en las siguientes metas relacionadas con el diseño de la rúbrica socioformativa: 1) mejorar la redacción y la pertinencia con el apoyo de expertos; 2) determinar la validez de contenido, mediante el juicio de expertos; y 3) determinar la consistencia interna entre los ítems del Instrumento, a través de la aplicación a un grupo piloto.

\section{Metodología}

Para realizar el analisis de validez de contenido y confiabilidad del instrumento, se considera someterlo al proceso de juicio de expertos y aplicación a un grupo piloto, para este caso, estudiantes de la población a la que está dirigido el instrumento.

\subsection{Tipo de estudio}

Se realizó un estudio instrumental de validez de contenido y confiabilidad del instrumento rúbrica socioformativa para evaluar la gestión del conocimiento durante el desarrollo de proyectos formativos. Este tipo de estudio hace referencia a todos aquellos procesos en donde existe un desarrollo de pruebas e instrumentos, que van desde el diseño y hasta su adaptación a las condiciones reales necesarias. (Montero \& León, 2007), para ello se realiza un análisis de contenido de los 8 items en función de la redacción y pertinencia con el apoyo de expertos, para el análisis de confiabilidad participaron 54 estudiantes, con 22 proyectos formativos, cada uno se realizó de manera colaborativa.

\subsection{Participantes}

El proceso de mejora en los ítems del instrumento, en relación con la validez de contenido: la redacción y pertinencia se hizo a través de juicio de expertos, en este caso trece (13) investigadores de la Escuelas Superior de Ingeniería Mecánica y Eléctrica (ESIME), Unidad Culhuacán (UC), del Instituto Politécnico Nacional (IPN) Ciudad de México, ellos fueron seleccionados en función de la experiencia que tienen en el diseño y validación de instrumentos y estudios relacionados en el área, tienen maestría y dos de ellas son doctoras en educación; su área de experiencia profesional es en docencia e investigación en el nivel superior y educación a distancia; el porcentaje de hombres que participó es de $37.5 \%$ y el de mujeres, $62.5 \%$. 
La población objetivo está conformada por 54 estudiantes, $94.4 \%$ hombres y $5.6 \%$ mujeres, estan inscritos en la carrera de Ingeniería en Comunicaciones y Electrónica (ICE) y en la carrea de Ingeniería en Computación (IC), de la ESIME UC del IPN, en los semestres: $2^{\circ}, 5^{\circ}, 6^{\circ}$ y $9^{\circ}$ en las asignaturas de Química Aplicada, Humanidades, Electrónica Lineal y Seminario y Proyecto de Ingeniería, respectivamente, en donde desarrollaron un proyecto formativo.

\subsection{Procedimiento}

El estudio de validez de contenido y confiabilidad del instrumento se llevó a cabo mediante las siguientes fases:

\subsubsection{Fase de diseño y revisión por expertos}

\section{Descripción del instrumento}

La rúbrica socioformativa se diseñó como instrumento para evaluar el proceso de la gestión del conocimiento durante el desarrollo de proyectos formativos. El diseño tiene una estructura tipo Likert; contiene 8 ítems que fueron redactados considerando la taxonomía socioformativa en la redacción de los indicadores y los niveles de desempeño; mismos que están acordes con tres dimensiones: 1) identificación de los requerimientos de información; 2) estrategias para el procesamiento de la información y la tercera dimensión; 3) aplicación de conocimiento con calidad y pertinencia para resolver el problema de contexto. Los ítems recaban información en función de las habilidades y destrezas, actitudes y aptitudes que los estudiantes del área de ingeniería deben demostrar y aplicar en el proceso de la gestión del conocimiento (constructo), durante el desarrollo de proyectos formativos. (ver tabla 1).

Tabla 1

Dimensiones e ítems del Instrumento

\begin{tabular}{|c|c|}
\hline Dimensiones & Preguntas del instrumento \\
\hline \multirow[t]{3}{*}{$\begin{array}{l}\text { Dimensión } 1 \text {. Identificación de los requerimientos de } \\
\text { información. }\end{array}$} & $\begin{array}{l}\text { 1.- ¿En qué nivel los estudiantes identificaron las } \\
\text { necesidades de la información para resolver e } \\
\text { problema de contexto? }\end{array}$ \\
\hline & $\begin{array}{l}\text { 2.- ¿En qué nivel el informe escrito contiene citas y } \\
\text { referencias actuales de la información propuesta } \\
\text { para resolver el problema de contexto? }\end{array}$ \\
\hline & $\begin{array}{l}\text { 3.- ¿En qué nivel en el informe escrito se integra la } \\
\text { lista de referencias bibliográficas de fuentes de } \\
\text { información confiable y especializada para resolver } \\
\text { el problema de contexto? }\end{array}$ \\
\hline \multirow[t]{3}{*}{$\begin{array}{l}\text { Dimensión 2. Estrategias para el Procesamiento de la } \\
\text { información. }\end{array}$} & $\begin{array}{l}\text { 4.- ¿En qué nivel los estudiantes aplicaron } \\
\text { estrategias de selección y sistematización de la } \\
\text { información? }\end{array}$ \\
\hline & $\begin{array}{l}\text { 5.- ¿En qué nivel los estudiantes emplean } \\
\text { estrategias para el trabajo colaborativo? }\end{array}$ \\
\hline & $\begin{array}{l}\text { 6.- ¿En qué nivel los estudiantes presentan } \\
\text { evidencias de trabajo colaborativo, realizado de } \\
\text { manera presencial y a distancia para resolver el } \\
\text { problema de contexto? }\end{array}$ \\
\hline \multirow[t]{2}{*}{$\begin{array}{l}\text { Dimensión } 3 \text {. Aplicación de conocimiento con calidad y } \\
\text { pertinencia para resolver el problema. }\end{array}$} & $\begin{array}{l}\text { 7.- ¿En qué nivel el conocimiento se aplicó con } \\
\text { objetividad y pertinencia en la resolución del } \\
\text { problema? }\end{array}$ \\
\hline & $\begin{array}{l}\text { 8.- ¿En qué nivel los estudiantes gestionan } \\
\text { conocimiento pertinente para resolver el problema } \\
\text { de contexto? }\end{array}$ \\
\hline
\end{tabular}

Fuente: Elaboración propia, adaptada de

Ortega-Carbajal, et al., (2015), Tobón (2014). 


\section{Revisión de expertos}

En este primer momento, la función de los expertos consiste en revisar de manera general tanto el orden y ubicación de los ítems en las diferentes dimensiones que tiene el instrumento y recomendar mejoras, como producto de la revisión. Participaron cuatro expertas (ver tabla 2) que tienen experiencia en el diseño de instrumentos y área afines.

Tabla 2

Datos Sociodemográficos de los Expertos

\begin{tabular}{|c|c|c|}
\hline \multirow[t]{3}{*}{ Expertos: 4} & Sexo & $100 \%$ mujeres \\
\hline & \multirow[t]{2}{*}{ Roles: } & Docentes: 3 \\
\hline & & Directivo: 1 \\
\hline & \multirow[t]{2}{*}{ Último nivel de estudio: } & Maestría: 2 \\
\hline & & Doctorado: 2 \\
\hline & \multirow{5}{*}{ Áreas de experiencia profesional: } & Docencia e Investigación, \\
\hline & & Docencia e Investigación \\
\hline & & educativa en el nivel superior, \\
\hline & & Industria Química, Educación a \\
\hline & & distancia e investigación. \\
\hline & Número de años de experiencia profesional: & Entre 15 y 38 \\
\hline & Número de artículos publicados en el área: & Entre 1 y 8 \\
\hline & Número de ponencias presentadas en el área: & entre 1 y 10 \\
\hline & Número de libros publicados en el área: & 1 \\
\hline & Experiencia en la revisión, diseño y/o & $100 \%$ de las participantes. \\
\hline & $\begin{array}{l}\text { validación de un determinado instrumento de } \\
\text { investigación: }\end{array}$ & \\
\hline
\end{tabular}

Fuente: Elaboración propia, adaptada de Tobón (2018)

\subsubsection{Fase de estudio: validez de contenido por juicio de expertos}

El método de juicio de expertos consiste en que un grupo de personas comprometidas, con experiencia en el diseño y validación de instrumentos, validen el contenido, hagan sugerencias y recomendaciones de mejora al instrumento. Como juez es necesario que validen el número de ítems que integran el instrumento, en función de los niveles de desempeño, desde nivel preformal y hasta el nivel estratégico en las dos categorías de análisis: Pertinencia y redacción; en la pertinencia, el ítem permite evaluar un aspecto central del propósito, constructo teórico y/o dimensiones del instrumento, para el caso de la redacción, permite evaluar, si el ítem es comprensible por los potenciales usuarios y cumple con las normas gramaticales de la lengua (Escobar-Pérez, \& Cuervo Martínez, 2008). Se comparte el enlace del instrumento diseñado en formularios de Google https://docs.google.com/forms/d/1pxeFyqZR_LopkkphQ1KAhEOselkRtRI2OzpjFNVy2N4/edit

Cuantitativamente se evaluó el instrumento, por medio del cálculo de la $\mathrm{V}$ de Aiken, que permitió la cuantificación de la relevancia de cada ítem, con respecto de un dominio de contenido por un número $\mathrm{x}$ de jueces, con valores desde 0 a 1, donde 1 indica la concordancia perfecta entre los jueces. Se aceptaron valores superiores a 0.8. Para los ítems que no alcanzaron estos valores, se corrigieron de acuerdo con las sugerencias de los jueces expertos (Aiken, 1980). 
Tabla 3

Datos sociodemográficos y académicos de los Jueces expertos

\begin{tabular}{|c|c|c|}
\hline \multirow[t]{9}{*}{ Jueces } & Sexo & $\begin{array}{l}\text { \% hombres } 15.38 \\
\% \text { mujeres } 84.62\end{array}$ \\
\hline & Roles: & Docentes: 13 \\
\hline & & Directivos: 0 \\
\hline & Último nivel de estudio: & $\begin{array}{l}\text { Maestría: } 13 \\
\text { Doctorado: } 9 \\
\text { Posdoctorado: } 0\end{array}$ \\
\hline & Áreas de experiencia profesional: & $\begin{array}{l}\text { Docencia e investigación, Ciencias } \\
\text { Sociales y humanidades, Ingeniería } \\
\text { en computación, Matemáticas, } \\
\text { Ingeniería Química, Educación a } \\
\text { distancia, Redes de datos, Docencia } \\
\text { y administración. }\end{array}$ \\
\hline & Número de años de experiencia profesional: & Desde 10 hasta 38 años \\
\hline & Número de artículos publicados en el área: & Desde 1 hasta 18 \\
\hline & Número de libros publicados en el área: & Desde 3 hasta 4 \\
\hline & $\begin{array}{l}\text { Experiencia en la revisión, diseño y/o } \\
\text { validación de un determinado instrumento } \\
\text { de investigación }\end{array}$ & $84.6 \%$ \\
\hline
\end{tabular}

Elaboración propia, adaptado del cuestionario de factores sociodemográficos, CIFE (2018).

\subsubsection{Fase: determinación de la confiabilidad por grupo piloto}

Para determinar la confiabilidad, el instrumento se aplicó a 54 estudiantes de la carrera de Ingeniería en Comunicaciones y Electrónica "ICE" de la Escuela Superior de Ingeniería Mecánica y Eléctrica "ESIME" Unidad Culhuacán "UC" del Instituto Politecnico Nacional "IPN", inscritos en las asignaturas: química aplicada, humanidades, electrónica lineal, seminario y proyecto de ingeniería, de los semestres $2^{\circ}, 5^{\circ}, 6^{\circ}, 8^{\circ}$ y $9^{\circ}$ respectivamente (grupo piloto). En este grupo de estudiantes durante su formación académica en la asignatura que se indica, se desarrollaron 22 proyectos en trabajo colaborativo (ver tabla 5). El propósito de aplicar la rúbrica socioformativa a este grupo es probarla a través de la evaluación del desempeño de la gestión del conocimiento de los alumnos en el desarrollo de un proyecto formativo. La rúbrica socioformativa fue aplicada en forma de encuesta mediante Google Forms:

https://docs.google.com/forms/d/1mEg01_djKFBb86gN0n8y1EHRYZJTufZzMRYgLhDC1Xw/edit

Tabla 4

Datos sociodemográficos del grupo piloto

\begin{tabular}{|c|c|c|}
\hline Grupo & Características & Datos \\
\hline \multirow[t]{9}{*}{ Grupo piloto } & Sexo & 51 hombres (94.4\%) \\
\hline & & 3 mujeres $(5.6 \%)$ \\
\hline & Promedio de edad en años & 23 \\
\hline & Zona de residencia & Ciudad de México \\
\hline & Promedio de años de estudio & 19 \\
\hline & Años de experiencia laboral & 0 \\
\hline & Condiciones económicas: & \%Bajas \\
\hline & & 100\%Media \\
\hline & & $\%$ Altas \\
\hline
\end{tabular}

Fuente: Elaborada propia, con datos sociodemográficos de los participantes.

Para la adecuación del instrumento a la población objetivo se consideró una encuesta de satisfacción que los participantes contestaron, hace referencia a 4 preguntas: 1) ¿cuál fue el grado de comprensión de las 
instrucciones del instrumento?; 2) ¿cuál fue el grado de comprensión de las preguntas o ítems?; 3) ¿cuál fue el grado de satisfacción con el instrumento?; 4) ¿cuál es el grado de relevancia de las preguntas? Se ofrecieron las siguientes opciones de respuesta: bajo grado, aceptable grado, buen grado y excelente grado, con esa encuesta se verificó el grado de satisfacción y relevancia que el instrumento representó para la población objetivo.

\section{Aspectos Éticos}

Considerando el código de ética del Instituto Politécnico Nacional (2004), en el capítulo IV.1 hace referencia a los docentes: "En función de la Integridad: "El professor deberá ser franco, honesto, cabal, recto, probo, justo y veraz en el cumplimiento de sus funciones y obligaciones" (p. 26). La Ley general de protección de datos en posesión de sujetos obligados (2018), "el Instituto Politecnico Nacional a través de sus unidades académicas y administrativas tienen la obligación de proteger los datos personales que manejen de conformidad con lo dispuesto por la ley general de datos personales en posesión de sujetos obligados y demás normatividad".

\section{Resultados y discusión}

Como producto del estudio, en la tabla 5, se muestra la rúbrica socioformativa para evaluar la gestión del conocimiento durante el desarrollo de proyectos formativos.

Tabla 5

Rúbrica Socioformativa para evaluar la gestión del conocimiento durante el desarrollo de proyectos formativos en estudiantes de ingeniería

\begin{tabular}{|c|c|}
\hline Criterios & Niveles de dominio \\
\hline $\begin{array}{l}\text { 1.- ¿En qué nivel los } \\
\text { estudiantes identificaron las } \\
\text { necesidades de la } \\
\text { información para resolver el } \\
\text { problema de contexto? }\end{array}$ & $\begin{array}{l}\text { 1.-NIVEL PREFORMAL. El informe escrito no está sustentado por información que lleve } \\
\text { a resolver el problema de contexto. } \\
\text { 2.-NIVEL RECEPTIVO. Los estudiantes buscaron información necesaria para resolver el } \\
\text { problema de contexto. } \\
\text { 3.-NIVEL RESOLUTIVO. Los estudiantes verificaron las necesidades de información para } \\
\text { resolver el problema de contexto. } \\
\text { 4.- NIVEL AUTÓNOMO. Los estudiantes identificaron los requerimientos de } \\
\text { información de manera autónoma para resolver el problema. } \\
\text { 5.- NIVEL ESTRATÉGICO. Los estudiantes proponen con claridad y con enfoque } \\
\text { multidisciplinar información para resolver el problema del contexto. }\end{array}$ \\
\hline $\begin{array}{l}\text { 2.- ¿En qué nivel el informe } \\
\text { escrito contiene citas y } \\
\text { referencias actuales de la } \\
\text { información propuesta para } \\
\text { resolver el problema de } \\
\text { contexto? }\end{array}$ & $\begin{array}{l}\text { 1. NIVEL PREFORMAL. En el informe escrito no se enumeran las referencias de las que } \\
\text { se obtiene la información para resolver el problema de contexto. } \\
\text { 2.- NIVEL RECEPTIVO. En el informe escrito se registran las citas y referencias de las } \\
\text { que se obtiene la información, pero estas son de más de } 15 \text { años atrás. } \\
\text { 3.-NIVEL RESOLUTIVO. En el informe escrito emplean citas y referencias de } \\
\text { información pertinentes de } 5 \text { años atrás para resolver el problema de contexto. } \\
\text { 4.-NIVEL AUTÓNOMO. En el informe escrito se aporta y se evalúa las citas y referencias } \\
\text { de información para que sean actuales, pertinentes y confiables para resolver el } \\
\text { problema de contexto. } \\
\text { 5.- NIVEL ESTRATÉGICO. En el informe se aplican estrategias creativas para innovar en } \\
\text { las referencias de información para que sean actuales, pertinentes y confiables para } \\
\text { resolver el problema de contexto. }\end{array}$ \\
\hline $\begin{array}{l}\text { 3.- ¿En qué nivel en el } \\
\text { informe escrito se integra la } \\
\text { lista de referencias } \\
\text { bibliográficas de fuentes de } \\
\text { información confiable y } \\
\text { especializada para resolver el } \\
\text { problema de contexto? }\end{array}$ & $\begin{array}{l}\text { 1. NIVEL PREFORMAL. En el informe escrito no se considera una lista de referencias de } \\
\text { información especializada para resolver el problema de contexto. } \\
\text { 2.- NIVEL RECEPTIVO. En la lista de referencias bibliográficas se recupera fuentes de } \\
\text { divulgación: vídeos o revistas de tipo "Muy interesante", para resolver el problema de } \\
\text { contexto. }\end{array}$ \\
\hline
\end{tabular}




\begin{tabular}{|c|c|}
\hline Criterios & Niveles de dominio \\
\hline & $\begin{array}{l}\text { 3.- NIVEL RESOLUTIVO. En la lista de referencias bibliográficas se cumple solo con } \\
\text { fuentes de información académica: obras de consulta como libros de texto } \\
\text { académicos, tesis y enciclopedias. } \\
\text { 4.- NIVEL AUTÓNOMO. La lista de referencias bibliográfica que presenta el informe } \\
\text { escrito está integrada principalmente por bibliografía de carácter científico y/o } \\
\text { especializado. } \\
\text { 5.- NIVEL ESTRATÉGICO. La lista referencias bibliográficas que presenta el informe } \\
\text { escrito está vinculada con información científica y/o especializada, así como manuales, } \\
\text { organizaciones, congresos, tratados y obras clásicas. }\end{array}$ \\
\hline $\begin{array}{l}\text { 4.- ¿En qué nivel los } \\
\text { estudiantes aplicaron } \\
\text { estrategias de selección y } \\
\text { sistematización de la } \\
\text { información? }\end{array}$ & $\begin{array}{l}\text { 1.NIVEL PREFORMAL. Aplicaron solo las estrategias para selección y sistematización de } \\
\text { la información indicadas por el profesor. } \\
\text { 2.NIVEL RECEPTIVO. Solo leyeron y transcribieron datos. } \\
\text { 3.NIVEL RESOLUTIVO. Propusieron un esquema específico. } \\
\text { 4. NIVEL AUTÓNOMO. Diseñaron e integraron información a partir de distintos } \\
\text { esquemas. } \\
\text { 5. NIVEL ESTRATÉGICO. Elaboraron cuadros comparativos, mapas conceptuales, } \\
\text { esquemas, fichas de trabajo, etc. }\end{array}$ \\
\hline $\begin{array}{l}\text { 5.- ¿En qué nivel los } \\
\text { estudiantes emplean } \\
\text { estrategias para el trabajo } \\
\text { colaborativo? }\end{array}$ & $\begin{array}{l}\text { 1.- NIVEL PREFORMAL. El trabajo se realizó dividiendo tareas entre el equipo. } \\
\text { 2.- NIVEL RECEPTIVO. Todo el trabajo colaborativo fue realizado en el tiempo de clase. } \\
\text { 3.- NIVEL RESOLUTIVO. Los estudiantes, además del trabajo colaborativo presencial, } \\
\text { ocuparon recursos tecnológicos, como es el correo electrónico, redes sociales y apps } \\
\text { entre otras para comunicarse a distancia. } \\
\text { 4.- NIVEL AUTÓNOMO. Los estudiantes compartieron y trabajaron información } \\
\text { presencial y a distancia a través de la nube. } \\
\text { 5.- NIVEL ESTRATÉGICO. Los estudiantes compartieron y trabajaron de manera } \\
\text { presencial, a través de la nube y por medio de herramientas de comunicación } \\
\text { multimedia como Skype, videoconferencia y facetime, ente otras. }\end{array}$ \\
\hline $\begin{array}{l}\text { 6.- ¿En qué nivel los } \\
\text { estudiantes presentan } \\
\text { evidencias de trabajo } \\
\text { colaborativo, realizado de } \\
\text { manera presencial y a } \\
\text { distancia para resolver el } \\
\text { problema de contexto? }\end{array}$ & $\begin{array}{l}\text { 1. NIVEL PREFORMAL. El equipo se desempeña con escasa colaboración y poca } \\
\text { coordinación. } \\
\text { 2. NIVEL RECEPTIVO. Los estudiantes solo describen las evidencias que hicieron en la } \\
\text { clase. } \\
\text { 3. NIVEL RESOLUTIVO. Los estudiantes emplean la computadora y el celular para } \\
\text { compartir mensajes que se enviaron relacionados con la información para la solución } \\
\text { del problema. } \\
\text { 4. NIVEL AUTÓNOMO. Los estudiantes aportan evidencias del trabajo que realizaron, } \\
\text { mostraron en la computadora los archivos de soporte, así como esquemas de } \\
\text { elaboración propia con la información encontrada para la solución del problema. } \\
\text { 5. NIVEL ESTRATÉGICO. Los estudiantes proponen medios de comunicación: Skype, } \\
\text { ZOOM, páginas académicas y blogs, para compartir las conferencias, las imágenes, los } \\
\text { mensajes de voz y datos que les sirvieron para resolver el problema de contexto. }\end{array}$ \\
\hline $\begin{array}{l}\text { 7.- ¿En qué nivel el } \\
\text { conocimiento se aplicó con } \\
\text { objetividad y pertinencia en } \\
\text { la resolución del problema? }\end{array}$ & $\begin{array}{l}\text { 1. NIVEL PREFORMAL. Los estudiantes aplicaron solo la información proporcionada por } \\
\text { el docente. } \\
\text { 2. NIVEL RECEPTIVO. Identificaron elementos adicionales para la solución del } \\
\text { problema, pero no los aplicaron. } \\
\text { 3.- NIVEL RESOLUTIVO. Los conceptos fueron aplicados de forma argumentada y } \\
\text { justificada con la teoría correspondiente para resolver el problema. } \\
\text { 4. NIVEL AUTÓNOMO. Aplicaron y expresaron pertinentemente el conocimiento en la } \\
\text { resolución del problema. } \\
\text { 5. NIVEL ESTRATÉGICO. El conocimiento apropiado por los estudiantes lo aplicaron } \\
\text { con calidad, pertinencia, eficiencia y eficacia para solucionar el problema. }\end{array}$ \\
\hline
\end{tabular}




\begin{tabular}{|c|c|}
\hline Criterios & Niveles de dominio \\
\hline $\begin{array}{l}\text { 8.- ¿̇n qué nivel los } \\
\text { estudiantes gestionan } \\
\text { conocimiento pertinente } \\
\text { para resolver el problema de } \\
\text { contexto? }\end{array}$ & $\begin{array}{l}\text { 1. NIVEL PREFORMAL. Los estudiantes acatan conocimientos dados por el docente } \\
\text { para resolver el problema de contexto. } \\
\text { 2. NIVEL RECEPTIVO. Los estudiantes describen fuentes documentales, en donde } \\
\text { encuentran conocimiento para resolver problemas de contexto. } \\
\text { 3. NIVEL RESOLUTIVO. Los estudiantes aplican estrategias didácticas para construir } \\
\text { conocimientos pertinentes para resolver el problema de contexto. } \\
\text { 4. NIVEL AUTÓNOMO. Los estudiantes analizan conceptos para gestionar } \\
\text { conocimiento pertinente para resolver el problema de contexto. } \\
\text { 5. NIVEL ESTRATÉGICO. Los estudiantes innovan en la construcción de conceptos para } \\
\text { aprender el conocimiento pertinente para resolver el problema de contexto. }\end{array}$ \\
\hline
\end{tabular}

Fuente: Elaboración propia

Del análisis cualitativo de los expertos se dejó ver de manera repetida aspectos de mejora en la redacción de los ítems y el uso de los verbos de acuerdo con la taxonomía socioformativa en donde se hizo ver que no había claridad y eran ambiguos. El análisis cuantitativo dejó ver que los valores de la $\mathrm{V}$ de Aiken para la pertinencia de los ítems resultaron mayores de 0.8 para el $88 \%$ de los ítems, el $12 \%$ de los ítems que resultaron con valores de la $\mathrm{V}$ de Aiken menor a 0.8 , fueron mejorados de acuerdo con las sugerencias de los jueces antes de ser aplicado el instrumento al grupo piloto. En la redacción de los ítems, la $V$ de Aiken resultó mayor de 0.8 en el 50\%, el 50\% de los ítems con valores de la $\mathrm{V}$ de Aiken menor a 0.8 se mejoraron en función de las recomendaciones de los jueces antes de ser aplicado el instrumento al grupo piloto.

Esta información puede ser consultada en el formulario de Google de la rúbrica Socioformativa:

https://docs.google.com/forms/d/1pxeFyqZR_LopkkphQ1KAhEOselkRtRI2OzpjFNVy2N4/edit

\subsection{Validación de la confiabilidad}

El tratamiento estadístico de la información recabada de la aplicación del instrumento al grupo piloto, se hizo con el software SPPS de donde resultó el cálculo del alfa de Cronbach >0.8 (ver tabla 6).

Se comparte el enlace para consultar la rúbrica socioformativa en Google Forms, en donde se llevó a cabo el proceso de aplicación del instrumento al grupo piloto.

https://docs.google.com/forms/d/1mEg01_djKFBb86gN0n8y1EHRYZJTufZzMRYgLhDC1Xw/edit\#responses

El estatus de satisfacción y aceptación del instrumento, por el grupo piloto, referente al grado de comprensión de las instrucciones del instrumento, el grado de comprensión de las preguntas o ítems, el grado de satisfacción con el instrumento y el grado de relevancia de las preguntas (Gráfico 1), se encuentra entre bueno y excelente. 
Tabla 6

Determinación del alfa de Cronbach con los resultados de aplicación del instrumento al grupo piloto usando el sotfware estadístico pssp

\begin{tabular}{|c|c|c|c|}
\hline \multicolumn{4}{|c|}{ Estadísticas de fiabilidad } \\
\hline Alfa de Cronbach & \multicolumn{2}{|c|}{$\begin{array}{l}\text { Alfa de Cronbach basada en } \\
\text { elementos estandarizados }\end{array}$} & $\mathrm{N}$ de eler \\
\hline .851 & \multicolumn{2}{|c|}{.857} & 8 \\
\hline \multicolumn{4}{|c|}{ Fuente: Programa estadístico SPSS versión 24} \\
\hline \multicolumn{4}{|c|}{ Estadísticas de elemento } \\
\hline & Media & $\begin{array}{l}\text { Desviación } \\
\text { estándar }\end{array}$ & $\mathrm{N}$ \\
\hline VAR00001 & 4.1818 & .95799 & 22 \\
\hline VAR00002 & 3.5455 & 1.22386 & 22 \\
\hline VAR00003 & 3.4091 & .95912 & 22 \\
\hline VAR00004 & 3.3636 & 1.25529 & 22 \\
\hline VAR00005 & 3.6364 & 1.25529 & 22 \\
\hline VAR00006 & 3.7273 & .76730 & 22 \\
\hline VAR00007 & 4.2727 & .98473 & 22 \\
\hline VAR00008 & 3.9091 & .75018 & 22 \\
\hline
\end{tabular}

Fuente: Programa estadístico SPSS versión 24

Gráfico 1

Resultado de la encuesta de satisfacción del instrumento por el grupo piloto (Grupo de estudiantes de la carrera de ICE de la ESIME UC del IPN).

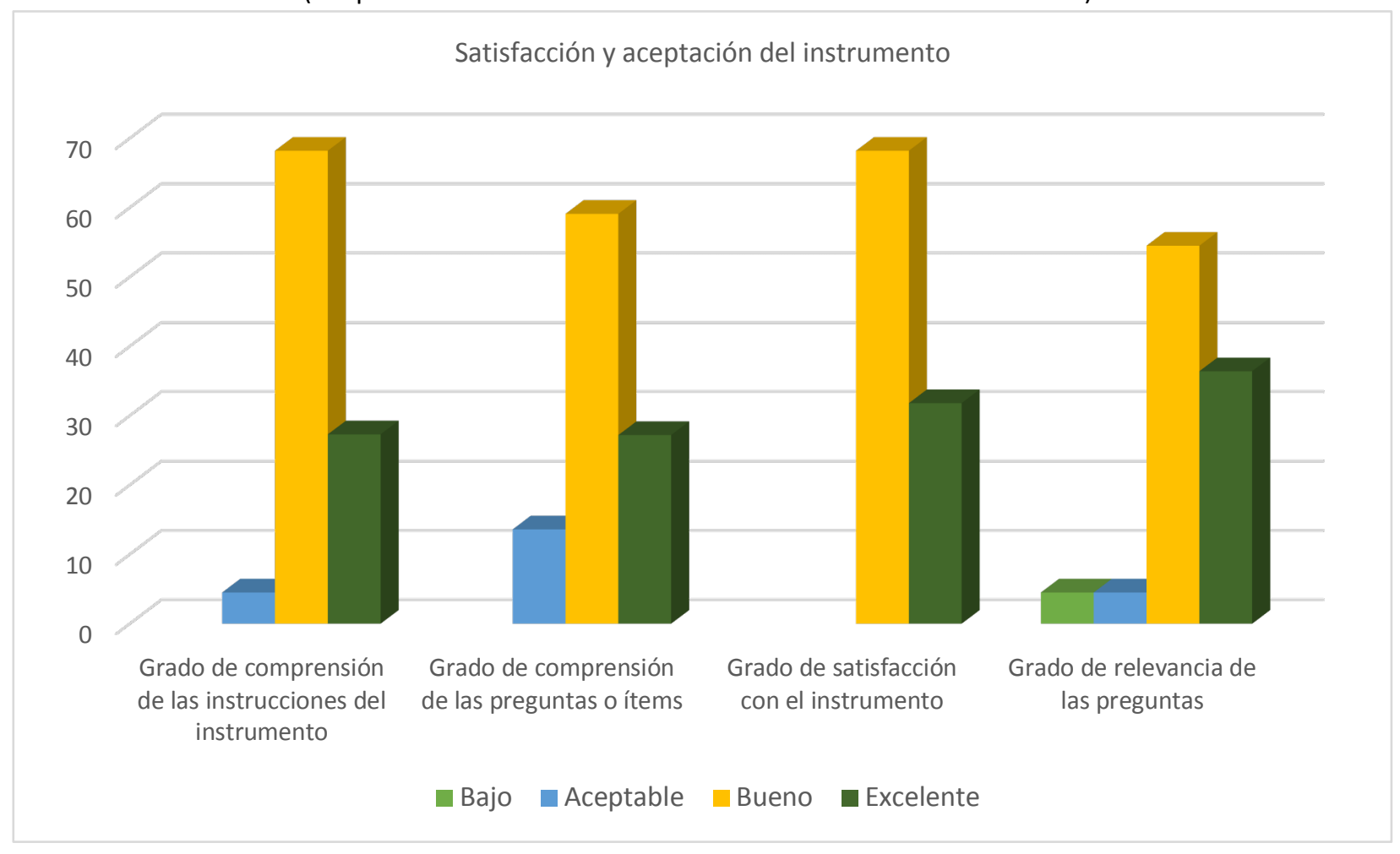

Fuente: Elaboración propia 


\section{Conclusiones}

Considerando las metas propuestas en esta investigación para el diseño y validación del instrumento y de acuerdo con los resultados cualitativos y cuantitativos que se tienen en el estudio realizado, la primera conclusión está basada en la confiabilidad de la rúbrica socioformativa que se diseñó para conocer el grado de dominio de la gestión del conocimiento durante el desarrollo de proyectos formativos por parte de estudiantes de nivel superior en el área de Ingeniería, debido a que tiene buena consistencia interna y los ítems están altamente correlacionados entre sí (Quero, 2010). El estudio muestra que el instrumento posee valores adecuados de confiabilidad, de acuerdo con Carmines y Zeller (1979), citado en Quero (2010) quienes establecen que valores de Alfa de Cronbach superiores a 0.8 significan un buen grado de consistencia interna en un instrumento, así también se tiene a Cronbach (1951) quien considera determinar la confiabilidad del instrumento en función de la proximidad entre los valores de la varianza y desviación estándar.

De acuerdo con lo descrito, es altamente recomendable que los docentes de escuelas de nivel superior del área de ingeniería, apliquen la rúbrica socioformativa como instrumento de evaluación (se deja el enlace en este documento), para evaluar el grado de domino y el desempeño de los estudiantes en el proceso de la gestión del conocimiento durante el desarrollo de proyectos formativos, dando oportunidad a los estudiantes de valorar sus logros y al docente de orientarlos a un proceso de mejora continua a través de la retroalimentación (Tobón, Pimienta, \& García, 2010). Pedrosa, Suárez-Álvarez y García-Cueto (2013) creen que un instrumento de medida es una forma de recuperación de información sobre una muestra de conductas de las que se quiere hacer ciertas inferencias.

En ese mismo contexto, es factible usar el instrumento en escuelas del área de Ingeniería, considerando el nivel de satisfacción y aceptación (que oscila en un rango de bueno a excelente) emitido por el grupo piloto en cuanto al grado de comprensión de las instrucciones del instrumento, en el grado de comprensión de las preguntas o ítems, en el grado de satisfacción con el instrumento y en el grado de relevancia de las preguntas.

Como segunda conclusión, solicitar apoyo de expertos en el área, con conocimientos y experiencia en el diseño y validación de instrumentos, con el propósito de revisar, validar y recibir sugerencias de mejora en el contenido de la rúbrica socioformativa, asegura mejor interpretación de la información en función de redacción y pertinencia, como se describe en Escobar-Pérez y Cuervo-Martínez (2008), el juicio de expertos es una práctica, que su importancia de aplicación radica en la interpretación de resultados de forma atinada y con eficiencia que permita la evaluación de la información obtenida de la revisión y que sea aplicada con la finalidad que se diseñó. Por su parte Cabero-Almenara y Llorente-Cejudo (2013) consideran que la revisión de los instrumentos por parte de expertos es una práctica que realiza un grupo de personas, quienes emiten un juicio o su opinión, sobre un aspecto en concreto, sobre un objeto en específico; para este caso sobre la redacción y pertinencia de los ítems del instrumento.

La tercera conclusión se enfoca en la validez de contenido, en la pertinencia y en la redacción del instrumento, Pedrosa, Suárez-Álvarez y García-Cueto (2013) refieren a la validez como una serie de pruebas y recolecta de datos que se hace para garantizar la pertinencia de las inferencias; en ese mimo sentido, Juárez - Hernández y Tobón (2017) la describen como la presencia de calidad en la precisión óptima en el instrumento. Para lograr esta característica en él, es primordial establecer la cantidad de jueces expertos, como señalan Escobar - Pérez y Cuervo - Martínez (2008) quienes citaron a Gable y Wolf (1993), Grant y Davis (1997) y Lynn (1996) en donde ellos proponen un rango de 2 y hasta 20 expertos y refieren la experiencia y los conocimientos en el área para que puedan participar en el proceso de validar en su contenido el instrumento.

En el contexto descrito en el párrafo anterior, se propone seguir realizando investigación en torno al área de oportunidad en la calidad del instrumento con miras en la validación del $50 \%$ de los ítems que se mejoraron con 
sugerencias de los jueces, se puede lograr considerando más investigaciones en el campo de la gestión del conocimiento, los proyectos formativos y la selección de la participación de los jueces de expertos, así como también compartir el conocimiento con otros docentes o investigadores en las áreas de investigación que se consideran en este trabajo.

\section{Referencias bibliográficas}

Cabero -Almenara, J. y Llorente- Cejudo, M. (2013). La aplicación de juicio de Expertos como técnica de evaluación de las tecnologías de la información (TIC). En Eduweb. Revista de tecnologías de la información y comunicación en Educación, 7(2),12 -12. Recuperado de http://www.riuc.bc.uc.edu.ve/bitstream/123456789/1175/1/art01.pdf

Cardona, S., Vélez, J. y Tobón, S. ( 2015). Proyectos Formativos y Evaluación con Rúbricas. Paradigma, 36(2), 74 -98. Recuperado de: http://ve.scielo.org/pdf/pdg/v36n2/art05.pdf

Cronbach, L.J. (1951). coefficient alpha and the internal structure of tests. Psychometrika 16(3). Recuperado de file:///E:/documentos\%20doctorado\%20cife/SEGUNDO\%20SEMESTRE/ARTICULOS\%20DE\%20VALIDEZ\%20 DE\%20CONTENIDO/ENVIADO\%2OPARA\%20EVALUACI\%C3\%93N/BF02310555.pdf

Escobar - Pérez, J., y Cuervo - Martínez, A. (2008). Validez de contenido y juicio de expertos: una aproximación a su utilización. En avances en medición, 27-36. Recuperado de http://www.humanas.unal.edu.co/psicometria/files/7113/8574/5708/Articulo3_Juicio_de_expertos_2736.pdf

Haynes, SN, Richard, DCS, y Kubany, ES (1995). Validez del contenido en la evaluación psicológica: un enfoque funcional de los conceptos y métodos. Evaluación psicológica, 7 (3), 238-247. http://dx.doi.org/10.1037/1040-3590.7.3.238

Hernández - Mosqueda, J., Tobón - Tobón, S., y Guerrero - Rosas, G. (2016). Hacia una evaluación integral del desempeño: las rúbricas socioformativas. Ra Ximhai, 12(6), 359-376. Recuperado de http://www.redalyc.org/articulo.oa?id=46148194025

Hernández-Mosqueda, J. (2013). Proceso de evaluación de las competencias desde la socioformación. Ra Ximhai, 9(4), 11-19. Recuperado de https://www.redalyc.org/pdf/461/46129004001.pdf

Ibarra-Piza, S., Segredo-Santamaría, S., Juárez-Hernández, L.G., y Tobón, S. (2018) Estudio de validez de contenido y confiabilidad de un instrumento para evaluar la metodología socioformativa en el diseño de cursos. Revista espacios, 39(53), 24. Recuperado de http://www.revistaespacios.com/cited2017/cited2017-24.html

IPN (2004) Código de ética del Instituto Politécnico Nacional, publicaciones de divulgación histórica. Recuperado de https://www.repositoriodigital.ipn.mx/handle/123456789/845

JS Hernández, JJ Vizcarra ( 2015). Didáctica para la formación integral en la sociedad del conocimiento. México: Horson Ediciones Escolares.

Juarez-Hernández, Tobón, S. ( 2018). Análisis de los elementos implícitos en la validación de contenido de un instrumento de investigación. Espacios, 39(53). Recuperado de http://www.revistaespacios.com/cited2017/cited2017-23.pdf

Montero, I. y León, O. G. (2007). Clasificación y descripción de las metodologías de investigación en Psicología, Vol 7(3), 847-862. Recuperado de: 
https://www.researchgate.net/publication/26420207_Clasificacion_y_descripcion_de_las_metodologias_ de_investigacion_en_Psicologia

Pedrosa, I., Suárez-Álvarez y García-Cueto, E. (2013). Evidencias sobre la Validez de Contenido: Avances Teóricos y Métodos para su Estimación. Acción Psicológica, 10(2), 10 - 20. http://dx.doi.org/10.5944/ap.10.2.11820

Quero Virla, M. (2010). Confiabilidad y coeficiente Alpha de Cronbach. Telos, 12(2), 248-252. Recuperado de https://www.redalyc.org/pdf/993/99315569010.pdf

SEP -IPN (2018) Aviso de privacidad integral alta, control y gestión del personal del IPN. Recuperado de https://www.ipn.mx/assets/files/main/docs/avisos-privacidad/integral-control-gestion-personal.pdf

Tobón, S. (2009) El aprendizaje de competencias mediante proyectos formativos. Bogotá: CIFE

Tobón, S. (2011). El modelo de las competencias en la educación desde la socioformación. En A. Jaik y A. Barraza (Coords.). Competencias y educación. Miradas múltiples de una relación (p. 14-24). REDIE.

Tobón, S. (2013a). Evaluación de las competencias en la educación básica (2a - Ed.). México: Santillana.

Tobón, S. (2017) Metodología de elaboración de una rúbrica socioformativa. México: CIFE.

Tobón, S. (2017). Ejes esenciales de la sociedad del conocimiento y la socioformación. Mount Dora (USA): Kresearch. dx.doi.org/10.24944/isbn.978-1-945721-18-2 Educación

Tobón, S.; Pimienta, J. y García, J. (2010). Secuencias didácticas: Aprendizaje y evaluación de competencias. México: Pearson.

Urrutia, M., Barrios, S., Gutiérrez, M. y Mayorga, M. (2014). Métodos óptimos para determinar validez de contenido. Scielo, 28(3),547-558.

Esta obra está bajo una Licencia Creative Commons

Attribución-NoCommercial 4.0 International

\section{(cc) EY-NC}

